$\frac{1}{2 \cdot 89}\left(n^{4}+1\right)$ for $n=2747,2771,2885$.

$\frac{1}{2 \cdot 97}\left(n^{4}+1\right)$ for $n=2669,2683,2749$.

New factorizations are as follows:

$$
\begin{aligned}
938^{4}+1 & =809273 \cdot 956569 \\
1060^{4}+1 & =847577 \cdot 1489513 \\
1348^{4}+1 & =940169 \cdot 3511993 \\
1512^{4}+1 & =926617 \cdot 5640361 \\
1874^{4}+1 & =914561 \cdot 13485457 \\
2100^{4}+1 & =17 \cdot 873553 \cdot 1309601 \\
2838^{4}+1 & =868841 \cdot 74663657 \\
2908^{4}+1 & =41 \cdot 940369 \cdot 1854793 \\
\frac{1}{2}\left(1155^{4}+1\right) & =830233 \cdot 1071761 \\
\frac{1}{2}\left(1191^{4}+1\right) & =935353 \cdot 1075577 \\
\frac{1}{2}\left(1509^{4}+1\right) & =872369 \cdot 2971849 \\
\frac{1}{2}\left(2635^{4}+1\right) & =857569 \cdot 28107577 \\
\frac{1}{2}\left(2765^{4}+1\right) & =908353 \cdot 32173321 \\
\frac{1}{2}\left(2977^{4}+1\right) & =17 \cdot 809041 \cdot 2855393
\end{aligned}
$$

The following factorization was omitted from my original table [1]:

$$
\frac{1}{2}\left(2055^{4}+1\right)=17 \cdot 572233 \cdot 916633 .
$$

The least integers still incompletely factored correspond to $n=1038$ and 1229 , for even and odd values of $n$, respectively.

11 rue Jean Jaurès

Luxembourg

1. A. Gloden, "Table de factorisation des nombres $n^{4}+1$ dans l'intervalle $1000<n<$ 3000," Institut Grand-Ducal de Luxembourg, Archives, Tome XVI, Luxembourg, 1946, p. 7188.

2. A. Gloden, Table des Solutions de la Congruence $x^{4}+1 \equiv 0(\bmod p)$ pour $800,000<p$ $<1,000,000$, published by the author, rue Jean Jaures, 11, Luxembourg, 1959.

\title{
A Note on the Solution of Quartic Equations
}

\section{By Herbert E. Salzer}

For any quartic equation with real coefficients,

$$
X^{4}+A X^{3}+B X^{2}+C X+D=0,
$$

the following condensation of the customary algebraic solution is recommended as quickest and easiest for the computer to follow (no mental effort required). It works in every exceptional case.

Received December 22, 1959. 
Denote the four roots of (1), by $X_{1}, X_{2}, X_{3}$, and $X_{4}$. With the aid of [1], solve the "resolvent cubic equation" $a x^{3}+b x^{2}+c x+d=0$ for the real root $x_{1}$ only, where

$$
a=1, \quad b=-B, \quad c=A C-4 D, \quad \text { and } \quad d=D\left(4 B-A^{2}\right)-C^{2} .
$$

Find

$$
m=+\sqrt{\frac{1}{4} A^{2}-B+x_{1}}, \quad n=\frac{A x_{1}-2 C}{4 m} .
$$

If $m=0$, take $n=\sqrt{\frac{1}{4} x_{1}{ }^{2}-D}$ and proceed according to the following Case $I$ or Case $I I$, depending upon whether $m$ is real or imaginary.

Case I: If $m$ is real, let $\left(\frac{1}{2} A^{2}-x_{1}-B\right)=\alpha, 4 n-A m=\beta, \sqrt{\alpha+\beta}=\gamma$, $\sqrt{\alpha-\beta}=\delta$, and finally

$$
\begin{aligned}
& X_{1}=\frac{-\frac{1}{2} A+m+\gamma}{2}, \quad X_{2}=\frac{-\frac{1}{2} A-m+\delta}{2}, \\
& X_{3}=\frac{-\frac{1}{2} A+m-\gamma}{2}, \text { and } \quad X_{4}=\frac{-\frac{1}{2} A-m-\delta}{2} .
\end{aligned}
$$
Let

Case $I I$ : If $m$ is imaginary, say $m=i m^{\prime}$, then $n$ is also imaginary, say $n=i n^{\prime}$.

$$
\begin{aligned}
\left(\frac{1}{2} A^{2}-x_{1}-B\right)=\alpha, \quad 4 n^{\prime}-A m^{\prime}=\beta, \quad+\sqrt{\alpha^{2}+\beta^{2}} & =\rho, \\
\sqrt{\frac{\alpha+\rho}{2}} & =\gamma, \quad \frac{\beta}{2 \gamma}=\delta,
\end{aligned}
$$

and finally

$$
\left\{\begin{array}{l}
X_{1}=\frac{-\frac{1}{2} A+\gamma+i\left(m^{\prime}+\delta\right)}{2}, \\
X_{3}=\frac{-\frac{1}{2} A-\gamma+i\left(m^{\prime}-\delta\right)}{2} \\
\text { and } X_{4}=\bar{X}_{3} \text {, the complex conjugate of } X_{1},
\end{array}\right.
$$

If $\gamma=0$, we must have $\alpha=-\alpha^{\prime}, \alpha^{\prime} \geqq 0$, and formula (4II) still holds provided that in it we replace $\delta$ by $+\sqrt{\alpha^{\prime}}$.

As an example consider the quartic equation $X^{4}+X^{3}+X^{2}+X+1=0$, where $A=B=C=D=1$, so that from (2) the resolvent cubic equation is $x^{3}-x^{2}-3 x+2=0$. From [1] we find $x_{1}=0.61803400$. From (3), $m=$ $+\sqrt{-0.13196600}=+0.36327125 i$, so that $m^{\prime}=+0.36327125$. Then $n=$ $\frac{-1.38196600}{1.45308500 i}=+0.95105655 i$, so that $n^{\prime}=+0.95105655$. Proceeding according to Case $I I, \alpha=-1.11803400, \beta=3.44095495, \rho=3.6180341, \gamma=1.1180340$ and $\delta=1.53884$ 18. Then from (4II) we obtain $X_{1}=0.3090170+0.9510565 i$, $X_{2}=\bar{X}_{1}=0.3090170-0.9510565 i, X_{3}=-0.8090170-0.5877853 i$ and 
$X_{4}=\bar{X}_{3}=-0.8090170+0.5877853 i$. These roots may be verified as correct, since they are known to be the complex fifth roots of unity, namely $X_{1}=\cos 72^{\circ}+$ $i \sin 72^{\circ}, X_{2}=\cos 288^{\circ}+i \sin 288^{\circ}, X_{3}=\cos 216^{\circ}+i \sin 216^{\circ}$, and $X_{4}=$ $\cos 144^{\circ}+i \sin 144^{\circ}$.

Convair Astronautics

San Diego, California

1. H. E. Salzer, C. H. Richards \& I. Arsham, Table for the Solution of Cubic Equations, McGraw-Hill, New York, 1958.

\section{A Conjugate Factor Method for the Solution of a Cubic}

\section{By D. A. Magula}

1. Introduction. This paper gives a simple method for computing the real roots of the reduced cubic equation with real coefficients,

$$
x^{3}+A x+B=0,
$$

having roots $a, b, c$. We assume $a$ to be real, since every cubic equation has at least one real root.

The method consists in factoring $B$, and setting one factor equal to $\pm \sqrt{m}$, the other $n$. For all pairs $m, n$ such that $m+n=-A, \pm \sqrt{m}$ is a root. If no such pair exists, a method of interpolation is shown.

2. Proof of Method. The reduced cubic equation (1) can be transformed, by using the relations between the roots and coefficients, into a complete cubic,

$$
p^{3}+6 A p^{2}+9 A^{2} p+4 A^{3}+27 B^{2}=0,
$$

where

$$
p=\left(-3 a^{2}-4 A\right) \text {. }
$$

Equation (2) can be written in the form:

$$
(p+A)^{2}(-p-4 A)=27 B^{2}
$$

or

$$
\frac{(p+A)}{3} \sqrt{\frac{(-p-4 A)}{3}}= \pm B
$$

Let

$$
\begin{gathered}
m=\frac{-p-4 A}{3} \text { and } n=\frac{p+A}{3} \\
n \sqrt{m}= \pm B
\end{gathered}
$$

and

$$
m+n=-A \text {. }
$$

Received September 21, 1959; in revised form, December 22, 1959. 